\title{
Un guide de présentation pour les aperçus
}

\author{
Correspondance : ccdr-rmtc@phac-aspc.gc.ca
}

Citation proposée : Un guide de présentation pour les aperçus. Relevé des maladies transmissibles au Canada 2016;42:200-1.

https://doi.org/10.14745/ccdr.v42i09a03f

Un aperçu est utile pour explorer un nouveau domaine, pour résumer l'état des données sur un sujet large et pour fournir des connaissances sur la progression d'un corpus de recherches. Parce que les données probantes en santé clinique et publique évoluent rapidement, un résumé et une évaluation préparés par un expert peuvent être extrêmement utiles. Par exemple, quels sont les défis de l'élimination de l'infection du VIH en pédiatrie (1)? Ou encore, quelles sont les données probantes actuelles concernant les maladies à prion, qui étaient considérées dans le passé comme étant extrêmement rares, mais qui sont maintenant liées à un groupe de désordres plus larges de protéines anormalement formées qui pourraient être plus communes que précédemment pensé (2)? Un aperçu par un expert de ce champ de recherche peut fournir une introduction utile aux questions émergentes, ainsi qu'un cadre pour mieux comprendre les développements subséquents (3).

Un aperçu n'est pas une ébauche d'examen systématique. L'examen systématique est idéal pour des sujets précis (4). Par exemple, un examen systématique est souvent utilisé pour analyser les preuves de l'efficacité d'un médicament précis pour une condition particulière au sein d'une population précise. Un aperçu est idéal pour des sujets généraux. Un examen systématique est une recherche qui examine des preuves en profondeur; un aperçu est une opinion d'experts, fondée sur des preuves, qui effleure l'ensemble des données probantes d'une perspective générale.

La faiblesse potentielle d'un aperçu est son biais. Les lecteurs ont besoin d'être rassurés que les données probantes résumées sont justes et exactes, et non injustement sélectives. Une recherche dans des multiples bases de données de documentation avec l'appui d'un bibliothécaire de recherche peut contribuer à répondre à ces préoccupations (5), tout comme une analyse transparente. Le but d'un aperçu n'est pas de fournir le niveau le plus élevé de preuve, mais bien de résumer, d'analyser et d'éclairer.

Parce que nous ne connaissons pas de lignes directrices pour la présentation d'un article d'aperçu, le Relevé des maladies transmissibles au Canada (RMTC) a élaboré une liste de vérification comportant 16 éléments fondée sur la documentation et les pratiques exemplaires dans les communications scientifiques. Cette liste de vérification définit le besoin d'aborder un sujet d'une manière qui est logique, équilibrée et approfondie, qui comprend une prise en compte des preuves contradictoires, des forces et des faiblesses, et des prochaines étapes potentielles (tableau 1).

Un aperçu est généralement d'une longueur de 1500 à 2000 mots. Comme pour toutes les soumissions, vérifier la section Renseignements à l'intention des auteurs du RTMC, publiée au début de chaque volume chaque année en janvier pour la préparation générale d'un article et les exigences de soumissions (6).
Tableau 1 : Liste de vérification pour les aperçus

\begin{tabular}{|c|c|c|}
\hline $\begin{array}{l}\text { Élément de la } \\
\text { déclaration }\end{array}$ & $\mathbf{N}^{\circ}$ & Description \\
\hline \multicolumn{3}{|l|}{ Titre/Résumé } \\
\hline Titre & 1 & $\begin{array}{l}\text { Donner un titre qui inclut la population, la } \\
\text { condition ou la question principale du sujet } \\
\text { de l'aperçu. }\end{array}$ \\
\hline Résumé & 2 & $\begin{array}{l}\text { Fournir un résumé de } 200 \text { à } 250 \text { mots qui } \\
\text { présente le sujet, pourquoi il est important, } \\
\text { l'objectif de l'aperçu, des éléments clés et } \\
\text { une conclusion. }\end{array}$ \\
\hline \multicolumn{3}{|l|}{ Introduction } \\
\hline $\begin{array}{l}\text { Présentation du } \\
\text { sujet }\end{array}$ & 3 & $\begin{array}{l}\text { Présenter le sujet de l'étude et expliquer } \\
\text { pourquoi il est important. }\end{array}$ \\
\hline $\begin{array}{l}\text { Justification de } \\
\text { l'étude }\end{array}$ & 4 & $\begin{array}{l}\text { Présenter la justification pour fournir un } \\
\text { aperçu. }\end{array}$ \\
\hline Objectif & 5 & Décrire clairement l'objectif de l'aperçu. \\
\hline \multicolumn{3}{|r|}{ 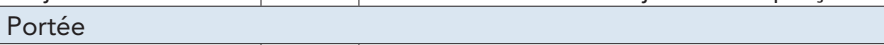 } \\
\hline $\begin{array}{l}\text { Environnement ou } \\
\text { population }\end{array}$ & 6 & $\begin{array}{l}\text { Décrire l'environnement ou les populations } \\
\text { sélectionnées de l'aperçu. }\end{array}$ \\
\hline Approche & 7 & $\begin{array}{l}\text { Présenter tout élément de décision sur ce } \\
\text { qui devrait être inclus ou ne pas être inclus } \\
\text { dans l'aperçu et sa justification. }\end{array}$ \\
\hline $\begin{array}{l}\text { Recherche } \\
\text { documentaire }\end{array}$ & 8 & $\begin{array}{l}\text { Présenter toutes les recherches } \\
\text { documentaires menées pour répondre à un } \\
\text { biais potentiel. }\end{array}$ \\
\hline \multicolumn{3}{|c|}{ Principales conclusions } \\
\hline $\begin{array}{l}\text { Population, } \\
\text { moment et lieu }\end{array}$ & 9 & $\begin{array}{l}\text { S'il y a lieu, présenter les résultats avec } \\
\text { suffisamment de détails pour donner une } \\
\text { idée de la population, du moment et du } \\
\text { lieu. }\end{array}$ \\
\hline $\begin{array}{l}\text { Analyse logique, } \\
\text { équilibrée et } \\
\text { approfondie }\end{array}$ & 10 & $\begin{array}{l}\text { Présenter une analyse qui démontre } \\
\text { clairement comment un aperçu répond à } \\
\text { l'objectif énoncé, est logique, comprend } \\
\text { des données probantes qui présentent } \\
\text { différents points de vue lorsqu'il est } \\
\text { indiqué de fournir une vue équilibrée, et } \\
\text { fournit une interprétation experte de la } \\
\text { documentation. }\end{array}$ \\
\hline $\begin{array}{l}\text { Soutien avec des } \\
\text { références }\end{array}$ & 11 & $\begin{array}{l}\text { Soutenir les affirmations et les faits avec les } \\
\text { références appropriées. }\end{array}$ \\
\hline \multicolumn{3}{|l|}{ Discussion } \\
\hline $\begin{array}{l}\text { Résumé des } \\
\text { principales } \\
\text { conclusions }\end{array}$ & 12 & $\begin{array}{l}\text { Résumer les principales conclusions } \\
\text { et préciser en quoi les résultats sont } \\
\text { pertinents en regard des objectifs de } \\
\text { l'étude. }\end{array}$ \\
\hline $\begin{array}{l}\text { Analyse } \\
\text { comparative }\end{array}$ & 13 & $\begin{array}{l}\text { Explorer des résultats qui corroborent } \\
\text { et prendre en compte des preuves } \\
\text { contradictoires (si disponible). }\end{array}$ \\
\hline Forces et faiblesses & 14 & $\begin{array}{l}\text { Présenter les forces et faiblesses de l'état } \\
\text { des connaissances sur le sujet de l'aperçu. }\end{array}$ \\
\hline Prochaines étapes & 15 & $\begin{array}{l}\text { Proposer d'autres étapes ou des domaines } \\
\text { de recherches à explorer à l'avenir. }\end{array}$ \\
\hline Conclusion & 16 & $\begin{array}{l}\text { Veiller à ce que la conclusion intègre les } \\
\text { principaux résultats et qu'elle réponde à } \\
\text { l'objectif de l'étude. }\end{array}$ \\
\hline
\end{tabular}

Abréviation : №, Nombre 


\section{Références}

1. Luzuriaga K, Mofenson LM. Challenges in the elimination of pediatric HIV-1 infection. N Engl J Med 2016;374:761-770 doi: 10.1056/NEJMra1505256.

2. Cashman NR. Propagation de protéines mal repliées : Nouvelles possibilités thérapeutiques, nouveau risque pour la santé publique. Relevé des maladies transmissibles au Canada 2015;41:226-230. http://www.phac-aspc. gc.ca/publicat/ccdr-rmtc/15vol41/dr-rm41-08/assets/ pdf/15vol41_08-fra.pdf.

3. Frontzek K, Lutz MI, Aguzzi A, Kovacs GG, Budka H. Amyloid- $\beta$ pathology and cerebral amyloid angiopathy are frequent in iatrogenic Creutzfeldt-Jakob disease after dural grafting. Swiss Med Wkly. 2016;146:w14287.
4. Guide de présentations des rapports d'examens systématiques. Relevé des maladies transmissibles au Canada 2015;41:87-91. http://www.phac-aspc. gc.ca/publicat/ccdr-rmtc/15vol41/dr-rm41-04/assets/ pdf/15vol41_04-fra.pdf.

5. Rethlefsen ML, Murad MH, Livingston EH. Engaging medical librarians to improve the quality of review articles. JAMA 2014;312(10):999-1000. doi:10.1001/jama.2014.9263.

6. Renseignements à l'intention des auteurs. Relevé des maladies transmissibles au Canada 2016;42:20-1. http:// www.phac-aspc.gc.ca/publicat/ccdr-rmtc/16vol42/drrm42-1/ar-04-fra.php.

\section{APPEL DE PRÉSENTATIONS}

\section{Avez-vous créé un nouveau programme intéressant dont les résultats s'avèrent prometteurs?}

Décrivez votre travail et il pourrait faire l'objet d'une publication dans notre numéro du printemps 2017 sur la science de la mise en œuvre.

La date limite des soumissions est le 10 novembre 2016.

Pour obtenir des conseils sur l'article, veuillez visiter notre page Soumettre un article en ligne : phac-aspc.gc.ca/publicat/ccdr-rmtc/ index-fra.php

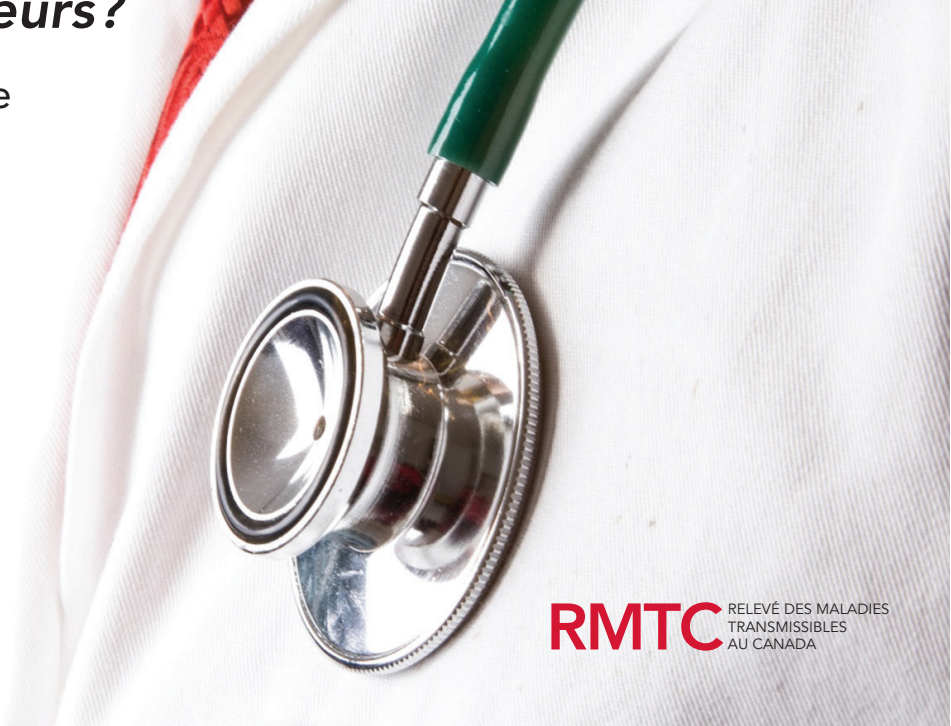

